\title{
Generalizations of the Markoff-Hurwitz equations over residue class rings
}

\author{
Ioulia N. Baoulina \\ Max Planck Institute for Mathematics \\ Vivatsgasse 7, 53111 Bonn, Germany \\ jbaulina@mail.ru
}

\begin{abstract}
In this paper, we use evaluations of Gauss sums modulo $p^{k}$ to derive expressions that allow us for a given generalized Markoff-Hurwitz equation to determine the number of its solutions over $\mathbb{Z} / p^{k} \mathbb{Z}$ if the number of solutions over $\mathbb{Z} / p \mathbb{Z}$ is known. We also calculate the corresponding Poincaré series.
\end{abstract}

Keywords: Generalized Markoff-Hurwitz equation; congruences in many variables; Dirichlet character; Gauss sum; Poincaré series; $Q$-conjecture.

Mathematics Subject Classification 2010: 11D79, 11G25

\section{Introduction}

Let $\mathcal{R}$ be a commutative ring. A Markoff-Hurwitz equation over $\mathcal{R}$ is an equation of the type

$$
x_{1}^{2}+\cdots+x_{n}^{2}=b x_{1} \cdots x_{n},
$$

where $b \in \mathcal{R} \backslash\{0\}$ and $n \geq 3$. Markoff [21] used continued fractions to find all integer solutions in the case $b=n=3$ and Hurwitz [18] described the set of integer solutions in the general case. For a history of the problem and related references see [5]. Baragar [6] and Silverman [22] studied solutions to a Markoff-Hurwitz equation when $\mathcal{R}$ is an order in a number field. Recently [4] we considered the case when $\mathcal{R}=\mathbb{Z} / p^{k} \mathbb{Z}$, where $p$ is a prime and $k$ is a positive integer. Using an elementary algebraic-combinatorial approach, we obtained expressions that allow us for a given Markoff-Hurwitz equation to find the number of its solutions over $\mathbb{Z} / p^{k} \mathbb{Z}$ if the number of solutions over $\mathbb{Z} / p \mathbb{Z}$ is known.

Carlitz [9] considered a generalized Markoff-Hurwitz equation

$$
a_{1} x_{1}^{2}+\cdots+a_{n} x_{n}^{2}=b x_{1} \cdots x_{n}+c,
$$


where $a_{1}, \ldots, a_{n}, b \in \mathcal{R} \backslash\{0\}, c \in \mathcal{R}$, over $\mathcal{R}=\operatorname{GF}(q)$ with odd $q$. He found the explicit formulas for the number of solutions when $n=3$ and when $n=4$ (under a certain restriction on the coefficients). Some generalizations of Carlitz's results can be found in $[2,3]$.

In this paper, we study a generalized Markoff-Hurwitz equation in the case when $\mathcal{R}=\mathbb{Z} / q \mathbb{Z}$, that is, we consider a congruence of the type

$$
a_{1} x_{1}^{2}+\cdots+a_{n} x_{n}^{2} \equiv b x_{1} \cdots x_{n}+c(\bmod q),
$$

where $n \geq 3, q>1$ is an integer and $a_{1}, \ldots, a_{n}, b, c$ are integers such that $\operatorname{gcd}\left(a_{1} \cdots a_{n}, q\right)=1$. Let $\bar{a}=\left(a_{1}, \ldots, a_{n}\right)$ and let $N_{q, n}(\bar{a}, b, c)$ denote the number of solutions to $(1.1)$ in $x_{1}, \ldots, x_{n}(\bmod q)$. For an integer $z$ and an odd $q$, let $(z / q)$ denote the generalized Jacobi symbol. Cohen [10] investigated (1.1) when $q$ is odd and $b \equiv 0(\bmod q)$. He proved [10, Corollary 1$]$ that

$$
N_{q, n}(\bar{a}, 0,0)= \begin{cases}q^{n-1} \sum_{d \mid q}\left(\frac{(-1)^{n / 2} a_{1} \cdots a_{n}}{d}\right) \frac{\varphi(d)}{d^{n / 2}} & \text { if } 2 \mid n, \\ q^{n-1} \sum_{d^{2} \mid q} \frac{\varphi\left(d^{2}\right)}{d^{n}} & \text { if } 2 \nmid n,\end{cases}
$$

where $\varphi$ is the Euler function. For the case $\operatorname{gcd}(c, q)=1$, Cohen [10, Corollary 2] gave the formula

$$
N_{q, n}(\bar{a}, 0, c)= \begin{cases}q^{n-1} \sum_{d \mid q}\left(\frac{(-1)^{n / 2} a_{1} \cdots a_{n}}{d}\right) \frac{\mu(d)}{d^{n / 2}} & \text { if } 2 \mid n, \\ q^{n-1} \sum_{d \mid q}\left(\frac{(-1)^{(n-1) / 2} a_{1} \cdots a_{n} c}{d}\right) \frac{\mu^{2}(d)}{d^{(n-1) / 2}} & \text { if } 2 \nmid n,\end{cases}
$$

where $\mu$ is the Möbius function.

Throughout much of this paper we are particularly interested in the congruence

$$
a_{1} x_{1}^{2}+\cdots+a_{n} x_{n}^{2} \equiv b x_{1} \cdots x_{n}+c\left(\bmod p^{k}\right),
$$

where $n \geq 3, p>2$ is a prime, $k$ is a positive integer, $a_{1}, \ldots, a_{n}, b, c$ are integers with $p \nmid a_{1} \cdots a_{n}$. From now on, we assume that $p \nmid c$. The result of Cohen mentioned above yields

$$
N_{p^{k}, n}(\bar{a}, 0, c)= \begin{cases}p^{k(n-1)}-\left(\frac{(-1)^{n / 2} a_{1} \cdots a_{n}}{p}\right) p^{((2 k-1)(n-1)-1) / 2} & \text { if } 2 \mid n, \\ p^{k(n-1)}+\left(\frac{(-1)^{(n-1) / 2} a_{1} \cdots a_{n} c}{p}\right) p^{(2 k-1)(n-1) / 2} & \text { if } 2 \nmid n .\end{cases}
$$


The special case

$$
N_{p, n}(\bar{a}, 0, c)= \begin{cases}p^{n-1}-\left(\frac{(-1)^{n / 2} a_{1} \cdots a_{n}}{p}\right) p^{(n-2) / 2} & \text { if } 2 \mid n, \\ p^{n-1}+\left(\frac{(-1)^{(n-1) / 2} a_{1} \cdots a_{n} c}{p}\right) p^{(n-1) / 2} & \text { if } 2 \nmid n,\end{cases}
$$

is due to Jordan [20] (see also [7, Theorem 10.5.1]). Carlitz [9, Theorem 1] showed that if $p \nmid b$ then

$$
\begin{aligned}
N_{p, 3}(\bar{a}, b, c)= & p^{2}+1+\left(\left(\frac{a_{1}}{p}\right)+\left(\frac{a_{2}}{p}\right)+\left(\frac{a_{3}}{p}\right)+\left(\frac{c}{p}\right)\right) \\
& \times\left(\frac{b^{2} c-4 a_{1} a_{2} a_{3}}{p}\right) p .
\end{aligned}
$$

Further, if $p \mid\left(b^{2} c^{2}-16 a_{1} a_{2} a_{3} a_{4}\right)$ then [9, Theorem 3] yields

$$
\begin{aligned}
N_{p, 4}(\bar{a}, b, c)= & p^{3}-1+\frac{1}{2}\left(\left(\frac{a_{1} a_{2}}{p}\right)+\left(\frac{a_{1} a_{3}}{p}\right)+\left(\frac{a_{1} a_{4}}{p}\right)+\left(\frac{a_{2} a_{3}}{p}\right)\right. \\
& \left.+\left(\frac{a_{2} a_{4}}{p}\right)+\left(\frac{a_{3} a_{4}}{p}\right)\right)\left(\frac{-1}{p}\right) p(p-2)-\left(\frac{-1}{p}\right) p \\
& -\left(\left(\frac{a_{1}}{p}\right)+\left(\frac{a_{2}}{p}\right)+\left(\frac{a_{3}}{p}\right)+\left(\frac{a_{4}}{p}\right)\right)\left(\frac{-2 c}{p}\right) p .
\end{aligned}
$$

Also, if $p \mid\left(b^{2} c^{2}-8 a_{1} a_{2} a_{3} a_{4}\right)$ and $\left(\frac{a_{1}}{p}\right)+\left(\frac{a_{2}}{p}\right)+\left(\frac{a_{3}}{p}\right)+\left(\frac{a_{4}}{p}\right)=0$ then $[9$, Theorem 2] together with properties of Jacobsthal sums [7, Proposition 6.1.10 and Theorem 6.2.1] imply that

$$
N_{p, 4}(\bar{a}, b, c)= \begin{cases}p^{3}-2 p-1 & \text { if } p \equiv 3(\bmod 4), \\ p^{3}+2(A+1) p-1 & \text { if } p \equiv 1(\bmod 4)\end{cases}
$$

where the integer $A$ is uniquely determined by

$$
p=A^{2}+B^{2}, \quad A \equiv-1(\bmod 4) .
$$

In general there is no explicit formula for evaluating $N_{p^{k}, n}(\bar{a}, b, c)$. The aim of this paper is to find expressions that allow us to calculate $N_{p^{k}, n}(\bar{a}, b, c)$ if $N_{p, n}(\bar{a}, b, c)$ is known. Our main results in Sec. 3 are Theorems 3.3-3.8, in which we obtain the desired expressions. In Sec. 4, we combine our expressions with the results of Carlitz mentioned above to determine explicitly $N_{p^{k}, n}(\bar{a}, b, c)$ and $N_{q, n}(\bar{a}, b, c)$ for $n=3$ and for $n=4$. In Sec. 5, we compute the corresponding Poincaré series and verify the $Q$-conjecture of Hayes and Nutt [17]. Poincaré series for more general polynomials are discussed in Sec. 6. 
Throughout this paper, we use the following notation. Let $N_{p^{k}, n}^{*}(\bar{a}, b, c)$ denote the number of solutions to (1.2) with $p \nmid x_{1} \cdots x_{n}$, and $N_{p^{k}, n}^{(0)}(\bar{a}, b, c)=N_{p^{k}, n}(\bar{a}, b, c)-$ $N_{p^{k}, n}^{*}(\bar{a}, b, c)$. Let $r$ be a non-negative integer such that

$$
p^{r} \|\left(b^{2} c^{n-2}-4(n-2)^{n-2} a_{1} \cdots a_{n}\right) .
$$

For $b^{2} c^{n-2}=4(n-2)^{n-2} a_{1} \cdots a_{n}$ we use the convention $r=\infty$. Let

$$
\theta= \begin{cases}\left(\frac{(-1)^{(n-2) / 2} \cdot 2(n-2)}{p}\right) & \text { if } 2 \mid n, \\ \left(\frac{(-1)^{(n-3) / 2} \cdot c(n-2)}{p}\right) & \text { if } 2 \nmid n .\end{cases}
$$

For any positive integer $q$, set $\zeta_{q}=\exp (2 \pi i / q)$.

\section{Preliminary Lemmas}

First we state our earlier result which will be useful in the sequel. We write $|\mathcal{A}|$ for the number of elements of a finite set $\mathcal{A}$.

Lemma 2.1. Let $f \in \mathbb{Z}\left[x_{1}, \ldots, x_{n}\right]$ be a nonzero polynomial, and let $k, \alpha_{1}, \ldots, \alpha_{n}$ be integers with $k \geq 2$ and $0 \leq \alpha_{1}, \ldots, \alpha_{n} \leq[k / 2]$. For $\nu \in\{k-1, k\}$, let $\mathcal{A}_{\bar{\alpha}, \nu}$ be the set of $n$-tuples $\left(u_{1}, \ldots, u_{n}\right)$ of integers such that $1 \leq u_{1}, \ldots, u_{n} \leq p^{\nu}, f\left(u_{1}, \ldots, u_{n}\right) \equiv 0$ $\left(\bmod p^{\nu}\right)$, and for each $j$,

$$
\begin{array}{c||l}
p^{\alpha_{j}} & \mid \frac{\partial f}{\partial x_{j}}\left(u_{1}, \ldots, u_{n}\right) \quad \text { if } \quad \alpha_{j}<[k / 2], \\
p^{\alpha_{j}} & \frac{\partial f}{\partial x_{j}}\left(u_{1}, \ldots, u_{n}\right) \quad \text { if } \quad \alpha_{j}=[k / 2] .
\end{array}
$$

Let

$$
\begin{aligned}
& \mathcal{A}_{\bar{\alpha}, \nu}^{(0)}=\left\{\left(u_{1}, \ldots, u_{n}\right) \in \mathcal{A}_{\bar{\alpha}, \nu}: p \mid u_{1} \cdots u_{n}\right\}, \\
& \mathcal{A}_{\bar{\alpha}, \nu}^{*}=\left\{\left(u_{1}, \ldots, u_{n}\right) \in \mathcal{A}_{\bar{\alpha}, \nu}: p \nmid u_{1} \cdots u_{n}\right\} .
\end{aligned}
$$

If $\min \left\{\alpha_{1}, \ldots, \alpha_{n}\right\}<[k / 2]$ then $\left|\mathcal{A}_{\bar{\alpha}, k}^{(0)}\right|=p^{n-1}\left|\mathcal{A}_{\bar{\alpha}, k-1}^{(0)}\right|$ and $\left|\mathcal{A}_{\bar{\alpha}, k}^{*}\right|=p^{n-1}\left|\mathcal{A}_{\bar{\alpha}, k-1}^{*}\right|$.

Proof. See [4, Lemma 2.1 and Remark 2.2].

Next we recall a few facts about characters. The following lemma gives the orthogonality relation for Dirichlet characters modulo $p^{k}$.

Lemma 2.2. For integers $x$ and $y$ with $p \nmid x$,

$$
\sum_{\chi\left(\bmod p^{k}\right)} \chi(x) \bar{\chi}(y)= \begin{cases}\varphi\left(p^{k}\right) & \text { if } p^{k} \mid(x-y), \\ 0 & \text { if } p^{k} \nmid(x-y),\end{cases}
$$

where the summation is taken over all Dirichlet characters $\chi$ modulo $p^{k}$. 
Proof. See [1, Theorem 6.16].

Since

$$
\sum_{\substack{\chi\left(\bmod p^{k}\right) \\ \chi-\operatorname{primitive}}} \chi(x) \bar{\chi}(y)=\sum_{\chi\left(\bmod p^{k}\right)} \chi(x) \bar{\chi}(y)-\sum_{\chi\left(\bmod p^{k-1}\right)} \chi(x) \bar{\chi}(y),
$$

for $k \geq 2$, the next lemma is a straightforward consequence of Lemma 2.2.

Lemma 2.3. Let $k \geq 2$. Then for integers $x$ and $y$ with $p \nmid x$,

$$
\sum_{\substack{\chi\left(\bmod p^{k}\right) \\ \chi-\text { primitive }}} \chi(x) \bar{\chi}(y)= \begin{cases}\varphi\left(p^{k}\right)-\varphi\left(p^{k-1}\right) & \text { if } p^{k} \mid(x-y), \\ -\varphi\left(p^{k-1}\right) & \text { if } p^{k-1} \|(x-y), \\ 0 & \text { if } p^{k-1} \nmid(x-y) .\end{cases}
$$

Let $\chi$ be a Dirichlet character modulo $p^{k}$. The Gauss sum corresponding to $\chi$ is defined by

$$
G(\chi)=\sum_{x=1}^{p^{k}} \chi(x) \zeta_{p^{k}}^{x}
$$

Gauss sums occur in the Fourier expansion of a primitive character.

Lemma 2.4. Let $\chi$ be a primitive Dirichlet character modulo $p^{k}$. Then for any integer $x$,

$$
\chi(x)=\frac{G(\chi)}{p^{k}} \sum_{y=1}^{p^{k}} \bar{\chi}(y) \zeta_{p^{k}}^{-x y}
$$

Proof. See [1, Theorem 8.20].

For a Dirichlet character $\chi$ modulo $p^{k}$, define

$$
T(\chi)=\sum_{1 \leq x_{1}, \ldots, x_{n} \leq p^{k}} \chi\left(x_{1}^{2} \cdots x_{n}^{2}\right) \bar{\chi}^{2}\left(a_{1} x_{1}^{2}+\cdots+a_{n} x_{n}^{2}-c\right) .
$$

In the following lemma we express $T(\chi)$ in terms of Gauss sums, under a certain restriction on the coefficients. For convenience, we also use the notation $\eta$ for the Jacobi symbol $(\dot{\bar{p}})$.

Lemma 2.5. Let $\chi$ be a primitive character modulo $p^{k}$. Assume that $\left(\frac{a_{1}}{p}\right)=\cdots=$ $\left(\frac{a_{n}}{p}\right)=\left(\frac{c(n-2)}{p}\right)$. Then

$$
T(\chi)=\frac{\bar{\chi}\left(a_{1} \cdots a_{n}\right) G\left(\bar{\chi}^{2}\right)}{p^{k}} \sum_{y=1}^{p^{k}} \bar{\chi}^{n-2}(y) \zeta_{p^{k}}^{-c y}\left(G(\chi)+\left(\frac{c(n-2) y}{p}\right) G(\chi \eta)\right)^{n} .
$$


Proof. Note that $\bar{\chi}^{2}$ is primitive. By Lemma 2.4

$$
\bar{\chi}^{2}\left(a_{1} x_{1}^{2}+\cdots+a_{n} x_{n}^{2}-c\right)=\frac{G\left(\bar{\chi}^{2}\right)}{p^{k}} \sum_{y=1}^{p^{k}} \chi^{2}(y) \zeta_{p^{k}}^{-\left(a_{1} x_{1}^{2}+\cdots+a_{n} x_{n}^{2}-c\right) y} .
$$

Hence

$$
\begin{aligned}
T(\chi) & =\frac{G\left(\bar{\chi}^{2}\right)}{p^{k}} \sum_{y=1}^{p^{k}} \chi^{2}(y) \zeta_{p^{k}}^{c y} \sum_{1 \leq x_{1}, \ldots, x_{n} \leq p^{k}} \chi\left(x_{1}^{2} \cdots x_{n}^{2}\right) \zeta_{p^{k}}^{-\left(a_{1} x_{1}^{2}+\cdots+a_{n} x_{n}^{2}\right) y} \\
& =\frac{G\left(\bar{\chi}^{2}\right)}{p^{k}} \sum_{\substack{p^{k}=1 \\
p \nmid y}} \chi^{2}(y) \zeta_{p^{k}}^{c y} \prod_{j=1}^{n} \sum_{x_{j}=1}^{p^{k}}\left(1+\left(\frac{x_{j}}{p}\right)\right) \chi\left(x_{j}\right) \zeta_{p^{k}}^{-a_{j} x_{j} y} \\
& =\frac{G\left(\bar{\chi}^{2}\right)}{p^{k}} \sum_{\substack{p^{k} \\
p \nmid y}} \chi^{2}(y) \zeta_{p^{k}}^{c y} \prod_{j=1}^{n} \bar{\chi}\left(-a_{j} y\right)\left(G(\chi)+\left(\frac{-a_{j} y}{p}\right) G(\chi \eta)\right),
\end{aligned}
$$

that is

$$
T(\chi)=\frac{\bar{\chi}\left(a_{1} \cdots a_{n}\right) G\left(\bar{\chi}^{2}\right)}{p^{k}} \sum_{y=1}^{p^{k}} \bar{\chi}^{n-2}(y) \zeta_{p^{k}}^{-c y}\left(G(\chi)+\left(\frac{c(n-2) y}{p}\right) G(\chi \eta)\right)^{n},
$$

as desired.

Let $k \geq 2$, and let $\psi$ be a primitive Dirichlet character modulo $p^{k}$ of order $\varphi\left(p^{k}\right)$ normalized such that

$$
\begin{aligned}
\psi\left(1+p^{k / 2}\right) & =\zeta_{p^{k / 2}}^{-1} & & \text { if } 2 \mid k, \\
\psi\left(1+p^{(k-1) / 2}+\frac{p+1}{2} p^{k-1}\right) & =\zeta_{p^{(k+1) / 2}}^{-1} & & \text { if } 2 \nmid k .
\end{aligned}
$$

Then every primitive character $\chi$ modulo $p^{k}$ has the form $\chi=\psi^{j}$ with $p \nmid j$.

Lemma 2.6. Let $k \geq 2$. For any integer $j$ with $p \nmid j$,

$$
G\left(\psi^{j}\right)= \begin{cases}p^{k / 2} \psi^{j}(j) \zeta_{p^{k}}^{j} & \text { if } 2 \mid k, \\ p^{k / 2} \psi^{j}(j)\left(\frac{j}{p}\right) \zeta_{p^{k}}^{j} \zeta_{8}^{1-p} & \text { if } 2 \nmid k .\end{cases}
$$

Proof. See [15, Corollary 2.1].

Lemma 2.7. Let $k \geq 2$. For any integer $j$ with $p \nmid j$,

$$
G\left(\psi^{j} \eta\right)=\left(\frac{j}{p}\right) G\left(\psi^{j}\right) .
$$


Proof. Observe that $\eta=\psi^{p^{k}(p-1) / 2}$. Applying Lemma 2.6, we deduce the asserted result.

Lemma 2.8. Let $k \geq 2$ and let $j$ be an integer with $p \nmid j$. Assume that $\left(\frac{a_{1}}{p}\right)=\cdots=$ $\left(\frac{a_{n}}{p}\right)=\left(\frac{c(n-2)}{p}\right)$. Then

$$
\begin{aligned}
T\left(\psi^{j}\right)= & 2^{n} p^{k n / 2} \psi^{j}\left(c^{n-2}\right) \bar{\psi}^{j}\left(4(n-2)^{n-2} a_{1} \cdots a_{n}\right) \\
& \times \begin{cases}1 & \text { if } 2 \mid k, \\
\left(\frac{2}{p}\right)^{n+1}\left(\frac{n-2}{p}\right)\left(\frac{j}{p}\right)^{n} i^{(n-2)(p-1)^{2} / 4} & \text { if } 2 \nmid k .\end{cases}
\end{aligned}
$$

Proof. Appealing to Lemmas 2.5 and 2.7, we obtain

$$
\begin{aligned}
T\left(\psi^{j}\right)= & \frac{\bar{\psi}^{j}\left(a_{1} \cdots a_{n}\right) G^{n}\left(\psi^{j}\right) G\left(\bar{\psi}^{2 j}\right)}{p^{k}} \sum_{y=1}^{p^{k}} \bar{\psi}^{j(n-2)}(y) \zeta_{p^{k}}^{-c y}\left(1+\left(\frac{j c(n-2) y}{p}\right)\right)^{n} \\
= & \frac{2^{n-1} \bar{\psi}^{j}\left(a_{1} \cdots a_{n}\right) G^{n}\left(\psi^{j}\right) G\left(\bar{\psi}^{2 j}\right)}{p^{k}} \\
& \times\left(\sum_{y=1}^{p^{k}} \bar{\psi}^{j(n-2)}(y) \zeta_{p^{k}}^{-c y}+\left(\frac{j c(n-2)}{p}\right) \sum_{y=1}^{p^{k}}\left(\frac{y}{p}\right) \bar{\psi}^{j(n-2)}(y) \zeta_{p^{k}}^{-c y}\right) \\
= & \frac{2^{n-1} \bar{\psi}^{j}\left(a_{1} \cdots a_{n}\right) \psi^{j(n-2)}(-c) G^{n}\left(\psi^{j}\right) G\left(\bar{\psi}^{2 j}\right)}{p^{k}} \\
& \times\left(G\left(\bar{\psi}^{j(n-2)}\right)+\left(\frac{j c(n-2)}{p}\right)\left(\frac{-c}{p}\right) G\left(\bar{\psi}^{j(n-2)} \eta\right)\right)
\end{aligned}
$$

that is

$$
T\left(\psi^{j}\right)=\frac{2^{n} \bar{\psi}^{j}\left(a_{1} \cdots a_{n}\right) \psi^{j(n-2)}(-c) G^{n}\left(\psi^{j}\right) G\left(\bar{\psi}^{2 j}\right) G\left(\bar{\psi}^{j(n-2)}\right)}{p^{k}} .
$$

Note that

$$
\begin{aligned}
\psi^{-2 j}(-2 j) & =\bar{\psi}^{j}(4) \psi^{-2 j}(j) \\
\psi^{-j(n-2)}(-j(n-2)) & =\bar{\psi}^{j(n-2)}(-1) \bar{\psi}^{j}\left((n-2)^{n-2}\right) \psi^{2 j}(j) \bar{\psi}^{j n}(j) .
\end{aligned}
$$

Combining these relations with Lemma 2.6 and (2.1) and using the fact that

$$
\zeta_{8}^{1-p}=\left(\frac{2}{p}\right) i^{(p-1)^{2} / 4},
$$

we deduce the desired result. 
Lemma 2.9. Let $k \geq 3$ be odd. Then for integers $x$ and $y$ with $p^{k-1} \mid(x-y)$ and $p \nmid x$,

$$
\sum_{j=1}^{\varphi\left(p^{k}\right)}\left(\frac{j}{p}\right) \psi^{j}(x) \bar{\psi}^{j}(y)=\left(\frac{-y}{p}\right)\left(\frac{(x-y) / p^{k-1}}{p}\right) i^{(p-1)^{2} / 4} p^{k-(3 / 2)}(p-1) .
$$

Proof. Since $p^{k-1} \mid(x-y)$, there exists a positive integer $t$ such that

$$
x \equiv y\left(1+p^{k-1} t\right) \equiv y\left(1+p^{k-1}\right)^{t} \quad\left(\bmod p^{k}\right)
$$

Note that

$$
\left(1+p^{(k-1) / 2}+\frac{p+1}{2} p^{k-1}\right)^{p^{(k-1) / 2}} \equiv 1+p^{k-1} \quad\left(\bmod p^{k}\right) .
$$

Thus, for any integer $j$,

$$
\psi^{j}(x) \bar{\psi}^{j}(y)=\psi^{j t p^{(k-1) / 2}}\left(1+p^{(k-1) / 2}+\frac{p+1}{2} p^{k-1}\right)=\zeta_{p^{(k+1) / 2}}^{-j t p^{(k-1) / 2}}=\zeta_{p}^{-j t} .
$$

Hence

$$
\sum_{j=1}^{\varphi\left(p^{k}\right)}\left(\frac{j}{p}\right) \psi^{j}(x) \bar{\psi}^{j}(y)=\sum_{j=1}^{\varphi\left(p^{k}\right)}\left(\frac{j}{p}\right) \zeta_{p}^{-j t}=\frac{\varphi\left(p^{k}\right)}{p}\left(\frac{-t}{p}\right) i^{(p-1)^{2} / 4} \sqrt{p}
$$

Since

$$
\frac{x-y}{p^{k-1}} \equiv y t \quad(\bmod p)
$$

we have

$$
\left(\frac{-t}{p}\right)=\left(\frac{-y}{p}\right)\left(\frac{(x-y) / p^{k-1}}{p}\right),
$$

and the asserted result follows.

An immediate consequence of Lemmas 2.3, 2.8 and 2.9 is the following (recall that $\theta$ was defined at the end of the introduction).

Lemma 2.10. Let $2 \leq k \leq r+1$. Assume that $\left(\frac{a_{1}}{p}\right)=\cdots=\left(\frac{a_{n}}{p}\right)=\left(\frac{c(n-2)}{p}\right)$ and $p \nmid b$. If $k n$ is even then

$$
\frac{1}{\varphi\left(p^{k}\right)} \sum_{\substack{\chi\left(\bmod p^{k}\right) \\ \chi \text { primitive }}} \chi\left(b^{2}\right) T(\chi)= \begin{cases}2^{n} \theta^{k} p^{(k n-2) / 2}(p-1) & \text { if } k \leq r \\ -2^{n} \theta^{r+1} p^{((r+1) n-2) / 2} & \text { if } k=r+1 .\end{cases}
$$

If $k n$ is odd then

$$
\frac{1}{\varphi\left(p^{k}\right)} \sum_{\substack{\chi\left(\bmod p^{k}\right) \\ \chi \text { primitive }}} \chi\left(b^{2}\right) T(\chi)=\left(\frac{\frac{b^{2} c^{n-2}-4(n-2)^{n-2} a_{1} \cdots a_{n}}{p^{k-1}}}{p}\right) \cdot 2^{n} \theta^{k} p^{(k n-1) / 2} .
$$




\section{Expressions for $N_{p^{k}, n}(\bar{a}, b, c)$}

First we show that the difference $N_{p^{k}, n}^{(0)}(\bar{a}, b, c)-p^{n-1} N_{p^{k-1}, n}^{(0)}(\bar{a}, b, c)$ vanishes for all $k \geq 2$.

Lemma 3.1. Let $k \geq 2$. Then $N_{p^{k}, n}^{(0)}(\bar{a}, b, c)-p^{n-1} N_{p^{k-1}, n}^{(0)}(\bar{a}, b, c)=0$.

Proof. For $\nu \in\{k-1, k\}$, let $\bar{N}_{p^{\nu}, n}^{(0)}(\bar{a}, b, c)$ denote the number of solutions to the congruence

$$
a_{1} x_{1}^{2}+\cdots+a_{n} x_{n}^{2} \equiv b x_{1} \cdots x_{n}+c\left(\bmod p^{\nu}\right)
$$

in $x_{1}, \ldots, x_{n}\left(\bmod p^{\nu}\right)$ such that $p \mid x_{1} \cdots x_{n}$ and

$$
\begin{aligned}
2 a_{1} x_{1} & \equiv b x_{2} x_{3} \cdots x_{n}\left(\bmod p^{[k / 2]}\right), \\
2 a_{2} x_{2} & \equiv b x_{1} x_{3} \cdots x_{n}\left(\bmod p^{[k / 2]}\right), \\
& \vdots \\
2 a_{n} x_{n} & \equiv b x_{1} x_{2} \cdots x_{n-1}\left(\bmod p^{[k / 2]}\right) .
\end{aligned}
$$

By Lemma 2.1

$$
N_{p^{k}, n}^{(0)}(\bar{a}, b, c)-p^{n-1} N_{p^{k-1}, n}^{(0)}(\bar{a}, b, c)=\bar{N}_{p^{k}, n}^{(0)}(\bar{a}, b, c)-p^{n-1} \bar{N}_{p^{k-1}, n}^{(0)}(\bar{a}, b, c) .
$$

It is readily seen that for each solution $\left(x_{1}, \ldots, x_{n}\right)$ to the system of congruences (3.2) with $p \mid x_{1} \cdots x_{n}$ we have $p\left|x_{1}, \ldots, p\right| x_{n}$. But since $p \nmid c$, none of these solutions satisfy (3.1). Therefore, $\bar{N}_{p^{k}, n}^{(0)}(\bar{a}, b, c)=\bar{N}_{p^{k-1}, n}^{(0)}(\bar{a}, b, c)=0$, and the result follows.

Next we show that in many cases $N_{p^{k}, n}^{*}(\bar{a}, b, c)-p^{n-1} N_{p^{k-1}, n}^{*}(\bar{a}, b, c)$ also vanishes.

Lemma 3.2. Let $k \geq 2$. Then $N_{p^{k}, n}^{*}(\bar{a}, b, c)-p^{n-1} N_{p^{k-1}, n}^{*}(\bar{a}, b, c)=0$ except possibly when $\left(\frac{a_{1}}{p}\right)=\cdots=\left(\frac{a_{n}}{p}\right)=\left(\frac{c(n-2)}{p}\right), p \nmid b$ and $k \leq r+1$.

Proof. For $\nu \in\{k-1, k\}$, let $\bar{N}_{p^{\nu}, n}^{*}(\bar{a}, b, c)$ denote the number of solutions to the congruence $(3.1)$ in $x_{1}, \ldots, x_{n}\left(\bmod p^{\nu}\right)$ such that $p \nmid x_{1} \cdots x_{n}$ and (3.2) holds. By Lemma 2.1

$$
N_{p^{k}, n}^{*}(\bar{a}, b, c)-p^{n-1} N_{p^{k-1}, n}^{*}(\bar{a}, b, c)=\bar{N}_{p^{k}, n}^{*}(\bar{a}, b, c)-p^{n-1} \bar{N}_{p^{k-1}, n}^{*}(\bar{a}, b, c) .
$$

Observe that $\bar{N}_{p^{k}, n}^{*}(\bar{a}, b, c)=\bar{N}_{p^{k-1}, n}(\bar{a}, b, c)=0$ for $b \equiv 0(\bmod p)$. Further, when $p \nmid x_{1} \cdots x_{n}$, the system of congruences (3.2) can be rewritten as

$$
2 a_{1} x_{1}^{2} \equiv \cdots \equiv 2 a_{n} x_{n}^{2} \equiv b x_{1} \cdots x_{n}\left(\bmod p^{[k / 2]}\right) .
$$

For any integers $x_{1}, \ldots, x_{n}$ with $p \nmid x_{1} \cdots x_{n}$ for which (3.1) and (3.4) hold simultaneously, we have

$$
(n-2) a_{j} x_{j}^{2} \equiv c\left(\bmod p^{[k / 2]}\right), \quad j=1, \ldots, n .
$$


Consequently, $\bar{N}_{p^{k}, n}^{*}(\bar{a}, b, c)=\bar{N}_{p^{k-1}, n}^{*}(\bar{a}, b, c)=0$ except possibly when $\left(\frac{a_{1}}{p}\right)=$ $\cdots=\left(\frac{a_{n}}{p}\right)=\left(\frac{c(n-2)}{p}\right)$ and $p \nmid b$.

Now assume that $\left(\frac{a_{1}}{p}\right)=\cdots=\left(\frac{a_{n}}{p}\right)=\left(\frac{c(n-2)}{p}\right), p \nmid b$ and $x_{1}, \ldots, x_{n}$ are integers with $p \nmid x_{1} \cdots x_{n}$ satisfying (3.1), (3.4) and (3.5). We have

$$
\left(a_{1} x_{1}^{2}+\cdots+a_{n} x_{n}^{2}-c\right)^{2} \equiv b^{2} x_{1}^{2} \cdots x_{n}^{2} \quad\left(\bmod p^{k-1}\right) .
$$

Multiplying both sides by $(n-2)^{n} a_{1} \cdots a_{n} \neq \equiv 0(\bmod p)$, we obtain

$$
\begin{gathered}
(n-2)^{n-2} a_{1} \cdots a_{n}\left(\sum_{j=1}^{n}\left((n-2) a_{j} x_{j}^{2}-c\right)+2 c\right)^{2} \\
\equiv b^{2} \prod_{j=1}^{n}\left(\left((n-2) a_{j} x_{j}^{2}-c\right)+c\right) \quad\left(\bmod p^{k-1}\right) .
\end{gathered}
$$

In view of (3.5),

$$
\left(\sum_{j=1}^{n}\left((n-2) a_{j} x_{j}^{2}-c\right)+2 c\right)^{2} \equiv 4 c^{2}+4 c \sum_{j=1}^{n}\left((n-2) a_{j} x_{j}^{2}-c\right) \quad\left(\bmod p^{k-1}\right)
$$

and

$$
\prod_{j=1}^{n}\left(\left((n-2) a_{j} x_{j}^{2}-c\right)+c\right) \equiv c^{n}+c^{n-1} \sum_{j=1}^{n}\left((n-2) a_{j} x_{j}^{2}-c\right) \quad\left(\bmod p^{k-1}\right) .
$$

We can now rewrite (3.6) in the equivalent form

$$
\left(b^{2} c^{n-2}-4(n-2)^{n-2} a_{1} \cdots a_{n}\right)\left(c^{2}+c \sum_{j=1}^{n}\left((n-2) a_{j} x_{j}^{2}-c\right)\right) \equiv 0 \quad\left(\bmod p^{k-1}\right) .
$$

By (3.5), this is only possible if $p^{k-1} \mid\left(b^{2} c^{n-2}-4(n-2)^{n-2} a_{1} \cdots a_{n}\right)$, or, equivalently, if $k \leq r+1$. Therefore we have established that $\bar{N}_{p^{k}, n}^{*}(\bar{a}, b, c)=\bar{N}_{p^{k-1}, n}^{*}(\bar{a}, b, c)=0$ except possibly when $\left(\frac{a_{1}}{p}\right)=\cdots=\left(\frac{a_{n}}{p}\right)=\left(\frac{c(n-2)}{p}\right), p \nmid b$ and $k \leq r+1$. The asserted result now follows from (3.3).

We are now ready to determine $N_{p^{k}, n}(\bar{a}, b, c)$ in the case $p \mid b$. We obtain immediately from (1.3) and Lemmas 3.1 and 3.2 the following.

Theorem 3.3. Assume that $p \mid b$. If $n$ is even then

$$
N_{p^{k}, n}(\bar{a}, b, c)=p^{k(n-1)}-\left(\frac{(-1)^{n / 2} a_{1} \cdots a_{n}}{p}\right) p^{((2 k-1)(n-1)-1) / 2} .
$$

If $n$ is odd then

$$
N_{p^{k}, n}(\bar{a}, b, c)=p^{k(n-1)}+\left(\frac{(-1)^{(n-1) / 2} a_{1} \cdots a_{n} c}{p}\right) p^{(2 k-1)(n-1) / 2} .
$$


Remark 3.4. Under the condition $p \mid b$ we have $N_{p^{k}, n}(\bar{a}, b, c)=N_{p^{k}, n}(\bar{a}, 0, c)$.

It remains to calculate the difference $N_{p^{k}, n}^{*}(\bar{a}, b, c)-p^{n-1} N_{p^{k-1}, n}^{*}(\bar{a}, b, c)$ in the case $\left(\frac{a_{1}}{p}\right)=\cdots=\left(\frac{a_{n}}{p}\right)=\left(\frac{c(n-2)}{p}\right), p \nmid b, 2 \leq k \leq r+1$.

Lemma 3.5. Let $2 \leq k \leq r+1$. Assume that $\left(\frac{a_{1}}{p}\right)=\cdots=\left(\frac{a_{n}}{p}\right)=\left(\frac{c(n-2)}{p}\right)$ and $p \nmid b$. If $n$ is even then

$$
N_{p^{k}, n}^{*}(\bar{a}, b, c)-p^{n-1} N_{p^{k-1}, n}^{*}(\bar{a}, b, c)= \begin{cases}2^{n-1} \theta^{k} p^{(k n-2) / 2}(p-1) & \text { if } k \leq r, \\ -2^{n-1} \theta^{r+1} p^{((r+1) n-2) / 2} & \text { if } k=r+1 .\end{cases}
$$

If $n$ is odd and $k \neq r+1$ then

$$
N_{p^{k}, n}^{*}(\bar{a}, b, c)-p^{n-1} N_{p^{k-1}, n}^{*}(\bar{a}, b, c)= \begin{cases}2^{n-1} p^{(k n-2) / 2}(p-1) & \text { if } 2 \mid k \text { and } k \leq r, \\ 0 & \text { otherwise. }\end{cases}
$$

If $n$ is odd, $r>0$ and $b^{2} c^{n-2} \neq 4(n-2)^{n-2} a_{1} \cdots a_{n}$ then

$$
\begin{aligned}
N_{p^{r+1}, n}^{*}(\bar{a}, b, c)-p^{n-1} N_{p^{r}, n}^{*}(\bar{a}, b, c)= & (-1)^{r}\left(\frac{\frac{b^{2} c^{n-2}-4(n-2)^{n-2} a_{1} \cdots a_{n}}{p^{r}}}{p}\right)^{r+1} \\
& \times 2^{n-1} \theta^{r+1} p^{[((r+1) n-1) / 2]} .
\end{aligned}
$$

Proof. For $\nu \in\{k-1, k\}$, let $\hat{N}_{p^{\nu}, n}^{*}(\bar{a}, b, c)$ denote the number of solutions to the congruence

$$
\left(a_{1} x_{1}^{2}+\cdots+a_{n} x_{n}^{2}-c\right)^{2} \equiv b^{2} x_{1}^{2} \cdots x_{n}^{2} \quad\left(\bmod p^{\nu}\right)
$$

in $x_{1}, \ldots, x_{n}\left(\bmod p^{\nu}\right)$ such that $p \nmid x_{1} \cdots x_{n}$. It is readily seen that

$$
\hat{N}_{p^{\nu}, n}^{*}(\bar{a}, b, c)=N_{p^{\nu}, n}^{*}(\bar{a}, b, c)+N_{p^{\nu}, n}^{*}(\bar{a},-b, c)=2 N_{p^{\nu}, n}^{*}(\bar{a}, b, c) .
$$

Further, by Lemma 2.2,

$$
\begin{aligned}
\hat{N}_{p^{k}, n}^{*}(\bar{a}, b, c) & \frac{1}{\varphi\left(p^{k}\right)} \sum_{\substack{1 \leq x_{1}, \ldots, x_{n} \leq p^{k} \\
p \nmid x_{1} \cdots x_{n}}} \sum_{\left(\bmod p^{k}\right)} \chi\left(b^{2} x_{1}^{2} \cdots x_{n}^{2}\right) \bar{\chi}^{2}\left(a_{1} x_{1}^{2}+\cdots+a_{n} x_{n}^{2}-c\right) \\
= & \frac{1}{\varphi\left(p^{k}\right)} \sum_{\substack{1 \leq x_{1}, \ldots, x_{n} \leq p^{k} \\
p \nmid x_{1} \cdots x_{n}}} \sum_{\chi\left(\bmod p^{k-1}\right)} \chi\left(b^{2} x_{1}^{2} \cdots x_{n}^{2}\right) \bar{\chi}^{2}\left(a_{1} x_{1}^{2}+\cdots+a_{n} x_{n}^{2}-c\right) \\
& +\frac{1}{\varphi\left(p^{k}\right)} \sum_{\substack{1 \leq x_{1}, \ldots, x_{n} \leq p^{k} \\
\chi\left(\bmod p^{k}\right) \\
\chi-\operatorname{primitive}}} \chi\left(b^{2} x_{1}^{2} \cdots x_{n}^{2}\right) \bar{\chi}^{2}\left(a_{1} x_{1}^{2}+\cdots+a_{n} x_{n}^{2}-c\right) \\
= & p^{n-1} \hat{N}_{p^{k-1}, n}^{*}(\bar{a}, b, c)+\frac{1}{\varphi\left(p^{k}\right)} \sum_{\substack{\chi\left(\bmod p^{k}\right) \\
\chi-\operatorname{primitive}}} \chi^{2}(b) T(\chi) .
\end{aligned}
$$


Hence

$$
N_{p^{k}, n}^{*}(\bar{a}, b, c)=p^{n-1} N_{p^{k-1}, n}^{*}(\bar{a}, b, c)+\frac{1}{2 \varphi\left(p^{k}\right)} \sum_{\substack{\chi\left(\bmod p^{k}\right) \\ \chi-\text { primitive }}} \chi\left(b^{2}\right) T(\chi)
$$

The required expressions now follow from Lemma 2.10.

Corollary 3.6. Let

$$
k \geq \begin{cases}3 \quad \text { if } 2 \mid n,\left(\frac{a_{1}}{p}\right)=\cdots=\left(\frac{a_{n}}{p}\right)=\left(\frac{c(n-2)}{p}\right) \text { and } \\ & b^{2} c^{n-2}=4(n-2)^{n-2} a_{1} \cdots a_{n}, \\ & \text { if } 2 \nmid n,\left(\frac{a_{1}}{p}\right)=\cdots=\left(\frac{a_{n}}{p}\right)=\left(\frac{c(n-2)}{p}\right) \text { and } \\ & b^{2} c^{n-2}=4(n-2)^{n-2} a_{1} \cdots a_{n}, \\ r+2 \quad & \text { if }\left(\frac{a_{1}}{p}\right)=\cdots=\left(\frac{a_{n}}{p}\right)=\left(\frac{c(n-2)}{p}\right) \text { and } \\ & b^{2} c^{n-2} \neq 4(n-2)^{n-2} a_{1} \cdots a_{n}, \\ & \text { otherwise. }\end{cases}
$$

If $2 \mid n,\left(\frac{a_{1}}{p}\right)=\cdots=\left(\frac{a_{n}}{p}\right)=\left(\frac{c(n-2)}{p}\right)$ and $b^{2} c^{n-2}=4(n-2)^{n-2} a_{1} \cdots a_{n}$ then

$$
N_{p^{k}, n}(\bar{a}, b, c)=\left(p^{n-1}+\theta p^{n / 2}\right) N_{p^{k-1}, n}(\bar{a}, b, c)-\theta p^{(3 n-2) / 2} N_{p^{k-2}, n}(\bar{a}, b, c) ;
$$

if $2 \nmid n,\left(\frac{a_{1}}{p}\right)=\cdots=\left(\frac{a_{n}}{p}\right)=\left(\frac{c(n-2)}{p}\right)$ and $b^{2} c^{n-2}=4(n-2)^{n-2} a_{1} \cdots a_{n}$ then

$$
N_{p^{k}, n}(\bar{a}, b, c)=p^{n-1} N_{p^{k-1}, n}(\bar{a}, b, c)+p^{n} N_{p^{k-2}, n}(\bar{a}, b, c)-p^{2 n-1} N_{p^{k-3}, n}(\bar{a}, b, c) ;
$$

otherwise

$$
N_{p^{k}, n}(\bar{a}, b, c)=p^{n-1} N_{p^{k-1}, n}(\bar{a}, b, c)
$$

Appealing to Lemmas 3.1, 3.2 and 3.5, we obtain the following results.

Theorem 3.7. Let $n$ be even and $p \nmid b$. If $\left(\frac{a_{1}}{p}\right)=\cdots=\left(\frac{a_{n}}{p}\right)=\left(\frac{c(n-2)}{p}\right)$ and $k \leq r$ then

$$
\begin{aligned}
N_{p^{k}, n}(\bar{a}, b, c)= & p^{(k-1)(n-1)} N_{p, n}(\bar{a}, b, c) \\
& +2^{n-1} p^{(k n-2) / 2}(p-1) \cdot \frac{p^{(k-1)(n-2) / 2}-\theta^{k-1}}{p^{(n-2) / 2}-\theta} .
\end{aligned}
$$




$$
\begin{aligned}
& \text { If }\left(\frac{a_{1}}{p}\right)=\cdots=\left(\frac{a_{n}}{p}\right)=\left(\frac{c(n-2)}{p}\right), r>0 \text { and } k>r \text { then } \\
& \qquad \begin{aligned}
N_{p^{k}, n}(\bar{a}, b, c)= & p^{(k-1)(n-1)} N_{p, n}(\bar{a}, b, c) \\
& +2^{n-1} p^{((2 k-r)(n-1)+r) / 2} \cdot \frac{p^{(r-1)(n-2) / 2}-\theta^{r-1}}{p^{(n-2) / 2}-\theta} \\
& -2^{n-1} p^{((2 k-r-1)(n-1)+r-1) / 2} \cdot \frac{p^{r(n-2) / 2}-\theta^{r}}{p^{(n-2) / 2}-\theta} .
\end{aligned}
\end{aligned}
$$

In all other cases

$$
N_{p^{k}, n}(\bar{a}, b, c)=p^{(k-1)(n-1)} N_{p, n}(\bar{a}, b, c) .
$$

Theorem 3.8. Let $n$ be odd and $p \nmid b$. If $\left(\frac{a_{1}}{p}\right)=\cdots=\left(\frac{a_{n}}{p}\right)=\left(\frac{c(n-2)}{p}\right)$ and $k \leq r$ then

$$
\begin{aligned}
& \left.\begin{array}{rl}
N_{p^{k}, n}(\bar{a}, b, c)= & p^{(k-1)(n-1)} N_{p, n}(\bar{a}, b, c) \\
& +2^{n-1} p^{k(n-1)-[k / 2](n-2)-1}(p-1) \cdot \frac{p^{[k / 2](n-2)}-1}{p^{n-2}-1} . \\
\text { If }\left(\frac{a_{1}}{p}\right)=\cdots=\left(\frac{a_{n}}{p}\right)=\left(\frac{c(n-2)}{p}\right), r \text { is odd and } k>r \text { then } \\
N_{p^{k}, n}(\bar{a}, b, c)= & p^{(k-1)(n-1)} N_{p, n}(\bar{a}, b, c) \\
& +2^{n-1} p^{((2 k-r+1)(n-1)+r-1) / 2} \cdot \frac{p^{(r-1)(n-2) / 2}-1}{p^{n-2}-1} \\
\text { If }\left(\frac{a_{1}}{p}\right)=\cdots= & \left(\frac{a_{n}}{p}\right)=\left(\frac{c(n-2)}{p}\right), r>0 \text { is even and } k>r \text { then } \\
N_{p^{k}, n}(\bar{a}, b, c)= & p^{(k-1)(n-1)} N_{p, n}(\bar{a}, b, c) \\
& +2^{n-1} p^{((2 k-r)(n-1)+r-2) / 2}(p-1) \cdot \frac{p^{r(n-2) / 2}-1}{p^{n-2}-1} \\
& +\left(\frac{\left.b^{2} c^{n-2}-4(n-2)\right)^{n-2} a_{1} \cdots a_{n}}{p^{r}}\right) \\
p
\end{array}\right) \cdot 2^{n-1} \theta p^{((2 k-r-1)(n-1)+r) / 2} .
\end{aligned}
$$

In all other cases

$$
N_{p^{k}, n}(\bar{a}, b, c)=p^{(k-1)(n-1)} N_{p, n}(\bar{a}, b, c)
$$

\section{Explicit Formulas for $N_{p^{k}, 3}(\bar{a}, b, c)$ and for $N_{p^{k}, 4}(\bar{a}, b, c)$}

In this section, we use the expressions obtained in the previous section together with the results of Carlitz [9] to determine explicitly $N_{p^{k}, n}(\bar{a}, b, c)$ for $n=3$ and for $n=4$. 
Combining (1.4) with Theorem 3.8 leads to the following.

Theorem 4.1. Let $p \nmid b$. If $\left(\frac{a_{1}}{p}\right)=\left(\frac{a_{2}}{p}\right)=\left(\frac{a_{3}}{p}\right)=\left(\frac{c}{p}\right)$ and $k \leq r$ then

$$
N_{p^{k}, 3}(\bar{a}, b, c)=p^{2 k}+4 p^{2 k-1}+p^{2 k-2}-4 p^{2 k-[k / 2]-1} .
$$

If $\left(\frac{a_{1}}{p}\right)=\left(\frac{a_{2}}{p}\right)=\left(\frac{a_{3}}{p}\right)=\left(\frac{c}{p}\right), r$ is odd and $k>r$ then

$$
N_{p^{k}, 3}(\bar{a}, b, c)=p^{2 k}+4 p^{2 k-1}+p^{2 k-2}-4 p^{2 k-((r+1) / 2)}-4 p^{2 k-((r+3) / 2)} .
$$

If $\left(\frac{a_{1}}{p}\right)=\left(\frac{a_{2}}{p}\right)=\left(\frac{a_{3}}{p}\right)=\left(\frac{c}{p}\right), r>0$ is even and $k>r$ then

$$
\begin{aligned}
N_{p^{k}, 3}(\bar{a}, b, c)= & p^{2 k}+4 p^{2 k-1}+p^{2 k-2} \\
& +\left(\left(\frac{\left(b^{2} c-4 a_{1} a_{2} a_{3}\right) c / p^{r}}{p}\right)-1\right) \cdot 4 p^{2 k-((r+2) / 2)}
\end{aligned}
$$

In all other cases

$$
\begin{aligned}
N_{p^{k}, 3}(\bar{a}, b, c)= & p^{2 k}+p^{2 k-2}+\left(\left(\frac{a_{1}}{p}\right)+\left(\frac{a_{2}}{p}\right)+\left(\frac{a_{3}}{p}\right)+\left(\frac{c}{p}\right)\right) \\
& \times\left(\frac{b^{2} c-4 a_{1} a_{2} a_{3}}{p}\right) p^{2 k-1} .
\end{aligned}
$$

Similarly, by combining (1.5) and (1.6) with Theorem 3.7, we arrive at the following results.

Theorem 4.2. Let $p \nmid b$. Assume that $p \mid\left(b^{2} c^{2}-16 a_{1} a_{2} a_{3} a_{4}\right)$. If $\left(\frac{a_{1}}{p}\right)=\left(\frac{a_{2}}{p}\right)=$ $\left(\frac{a_{3}}{p}\right)=\left(\frac{a_{4}}{p}\right)=\left(\frac{2 c}{p}\right), p \equiv 1(\bmod 4)$ and $k \leq r$ then

$$
N_{p^{k}, 4}(\bar{a}, b, c)=p^{3 k}+3 p^{3 k-1}-3 p^{3 k-2}-p^{3 k-3}-8 p^{2 k-1} .
$$

If $\left(\frac{a_{1}}{p}\right)=\left(\frac{a_{2}}{p}\right)=\left(\frac{a_{3}}{p}\right)=\left(\frac{a_{4}}{p}\right)=\left(\frac{2 c}{p}\right), p \equiv 3(\bmod 4)$ and $k \leq r$ then

$$
\begin{aligned}
N_{p^{k}, 4}(\bar{a}, b, c)= & p^{3 k}-3 p^{3 k-1}+11 p^{3 k-2}-p^{3 k-3} \\
& +8 p^{2 k-1}(p-1) \cdot \frac{p^{k-1}-(-1)^{k-1}}{p+1}
\end{aligned}
$$

If $\left(\frac{a_{1}}{p}\right)=\left(\frac{a_{2}}{p}\right)=\left(\frac{a_{3}}{p}\right)=\left(\frac{a_{4}}{p}\right)=\left(\frac{2 c}{p}\right), p \equiv 1(\bmod 4)$ and $k>r$ then

$$
N_{p^{k}, 4}(\bar{a}, b, c)=p^{3 k}+3 p^{3 k-1}-3 p^{3 k-2}-p^{3 k-3}-8 p^{3 k-r-1}-8 p^{3 k-r-2} .
$$

If $\left(\frac{a_{1}}{p}\right)=\left(\frac{a_{2}}{p}\right)=\left(\frac{a_{3}}{p}\right)=\left(\frac{a_{4}}{p}\right)=\left(\frac{2 c}{p}\right), p \equiv 3(\bmod 4)$ and $k>r$ then

$$
\begin{aligned}
N_{p^{k}, 4}(\bar{a}, b, c)= & p^{3 k}-3 p^{3 k-1}+11 p^{3 k-2}-p^{3 k-3} \\
& +8 p^{3 k-r} \cdot \frac{p^{r-1}-(-1)^{r-1}}{p+1}-8 p^{3 k-r-2} \cdot \frac{p^{r}-(-1)^{r}}{p+1} .
\end{aligned}
$$


In all other cases

$$
\begin{aligned}
N_{p^{k}, 4}(\bar{a}, b, c)= & p^{3 k}+\frac{1}{2}\left(\left(\frac{a_{1} a_{2}}{p}\right)+\left(\frac{a_{1} a_{3}}{p}\right)+\left(\frac{a_{1} a_{4}}{p}\right)+\left(\frac{a_{2} a_{3}}{p}\right)\right. \\
& \left.+\left(\frac{a_{2} a_{4}}{p}\right)+\left(\frac{a_{3} a_{4}}{p}\right)\right)\left(\frac{-1}{p}\right) p^{3 k-2}(p-2)-\left(\frac{-1}{p}\right) p^{3 k-2} \\
& -\left(\left(\frac{a_{1}}{p}\right)+\left(\frac{a_{2}}{p}\right)+\left(\frac{a_{3}}{p}\right)+\left(\frac{a_{4}}{p}\right)\right)\left(\frac{-2 c}{p}\right) p^{3 k-2}-p^{3 k-3} .
\end{aligned}
$$

Theorem 4.3. Let $p \nmid b$. Assume that $p \mid\left(b^{2} c^{2}-8 a_{1} a_{2} a_{3} a_{4}\right)$ and $\left(\frac{a_{1}}{p}\right)+\left(\frac{a_{2}}{p}\right)+\left(\frac{a_{3}}{p}\right)+$ $\left(\frac{a_{4}}{p}\right)=0$. Then

$$
N_{p^{k}, 4}(\bar{a}, b, c)=\left\{\begin{array}{lll}
p^{3 k}-2 p^{3 k-2}-p^{3 k-3} & \text { if } p \equiv 3 \quad(\bmod 4), \\
p^{3 k}+2(A+1) p^{3 k-2}-p^{3 k-3} & \text { if } p \equiv 1 \quad(\bmod 4),
\end{array}\right.
$$

where the integer $A$ is uniquely determined by (1.7).

Next we evaluate the number $N_{q, n}(\bar{a}, b, c)$ of solutions to (1.1), under a certain restriction on $q$. If $\operatorname{gcd}\left(a_{1} \cdots a_{n} c, q\right)=1$ and each prime divisor of $q$ divides $b$ then, by the remark following Theorem $3.3, N_{q, n}(\bar{a}, b, c)=N_{q, n}(\bar{a}, 0, c)$. Hence by [10, Corollary 2],

$$
N_{q, n}(\bar{a}, b, c)= \begin{cases}q^{n-1} \sum_{d \mid q}\left(\frac{(-1)^{n / 2} a_{1} \cdots a_{n}}{d}\right) \frac{\mu(d)}{d^{n / 2}} & \text { if } 2 \mid n, \\ q^{n-1} \sum_{d \mid q}\left(\frac{(-1)^{(n-1) / 2} a_{1} \cdots a_{n} c}{d}\right) \frac{\mu^{2}(d)}{d^{(n-1) / 2}} & \text { if } 2 \nmid n .\end{cases}
$$

Using Theorems 4.1-4.3, we can easily obtain expressions for $N_{q, n}(\bar{a}, b, c)$ in some other cases.

Theorem 4.4. Let $q>1$ be an odd integer coprime with $a_{1}, a_{2}, a_{3}$ and $c$. Write $q=$ $q_{1} q_{2} q_{3} q_{4} q_{5}$, where $q_{1}, q_{2}, q_{3}, q_{4}, q_{5}$ are pairwise coprime positive integers satisfying the following conditions:

(a) each prime divisor of $q_{1}$ divides $b$;

(b) $b$ and $q_{2} q_{3} q_{4} q_{5}$ are coprime;

(c) if $p$ is a prime dividing $q_{2}$ then $\left(\frac{a_{1}}{p}\right)=\left(\frac{a_{2}}{p}\right)=\left(\frac{a_{3}}{p}\right)=\left(\frac{c}{p}\right), p^{2} \mid q_{2}$ and $p \|\left(b^{2} c-4 a_{1} a_{2} a_{3}\right)$;

(d) $b^{2} c-4 a_{1} a_{2} a_{3}$ and $q_{3} q_{4} q_{5}$ are coprime;

(e) if $p$ is a prime dividing $q_{3} q_{4} q_{5}$ then

$$
\left(\frac{a_{1}}{p}\right)+\left(\frac{a_{2}}{p}\right)+\left(\frac{a_{3}}{p}\right)+\left(\frac{c}{p}\right)=\left(\frac{b^{2} c-4 a_{1} a_{2} a_{3}}{p}\right) \cdot\left\{\begin{aligned}
0 & \text { if } p \mid q_{3} \\
2 & \text { if } p \mid q_{4} \\
-2 & \text { if } p \mid q_{5}
\end{aligned}\right.
$$


Then

$$
\begin{aligned}
N_{q, 3}(\bar{a}, b, c)= & q^{2}\left(\sum_{d_{1} \mid q_{1}}\left(\frac{-a_{1} a_{2} a_{3} c}{d_{1}}\right) \frac{\mu^{2}\left(d_{1}\right)}{d_{1}}\right)\left(\sum_{d_{2} \mid q_{2}} \frac{\mu\left(d_{2}\right) 3^{\nu\left(d_{2}\right)}}{d_{2}^{2}}\right) \\
& \times\left(\sum_{d_{3} \mid q_{3}} \frac{\mu^{2}\left(d_{3}\right)}{d_{3}^{2}}\right)\left(\sum_{d_{4} \mid q_{4}} \frac{\mu^{2}\left(d_{4}\right)}{d_{4}}\right)^{2} \frac{\varphi^{2}\left(q_{5}\right)}{q_{5}^{2}}
\end{aligned}
$$

where $\nu\left(d_{2}\right)$ denotes the number of distinct prime divisors of $d_{2}$. In particular, if $\operatorname{gcd}(b, q)=\operatorname{gcd}\left(b^{2} c-4 a_{1} a_{2} a_{3}, q\right)=1$ and for each prime $p$ dividing $q$ we have $\left(\frac{a_{1}}{p}\right)+\left(\frac{a_{2}}{p}\right)+\left(\frac{a_{3}}{p}\right)+\left(\frac{c}{p}\right)=-2 \cdot\left(\frac{b^{2} c-4 a_{1} a_{2} a_{3}}{p}\right)$ then $N_{q, 3}(\bar{a}, b, c)=\varphi^{2}(q)$.

Theorem 4.5. Let $q>1$ be an odd integer coprime with $a_{1}, a_{2}, a_{3}, a_{4}$ and $c$. Write $q=q_{1} q_{2} q_{3} q_{4} q_{5}$, where $q_{1}, q_{2}, q_{3}, q_{4}, q_{5}$ are pairwise coprime positive integers satisfying the following conditions:

(a) each prime divisor of $q_{1}$ divides $b$;

(b) each prime divisor of $q_{2} q_{3}$ divides $b^{2} c^{2}-8 a_{1} a_{2} a_{3} a_{4}$;

(c) each prime divisor of $q_{4} q_{5}$ divides $b^{2} c^{2}-16 a_{1} a_{2} a_{3} a_{4}$;

(d) if $p$ is a prime dividing $q_{2} q_{3} q_{4}$ then $\left(\frac{a_{1}}{p}\right)+\left(\frac{a_{2}}{p}\right)+\left(\frac{a_{3}}{p}\right)+\left(\frac{a_{4}}{p}\right)=0$;

(e) if $p$ is a prime dividing $q_{2}$ then $p-1$ is a perfect square;

(f) if $p$ is a prime dividing $q_{3} q_{5}$ then $p \equiv 3(\bmod 4)$;

(g) if $p$ is a prime dividing $q_{5}$ then $\left(\frac{a_{1}}{p}\right)+\left(\frac{a_{2}}{p}\right)+\left(\frac{a_{3}}{p}\right)+\left(\frac{a_{4}}{p}\right)=-4 \cdot\left(\frac{2 c}{p}\right)$.

Then

$$
\begin{aligned}
N_{q, 4}(\bar{a}, b, c)= & q^{3}\left(\sum_{d_{1} \mid q_{1}}\left(\frac{a_{1} a_{2} a_{3} a_{4}}{d_{1}}\right) \frac{\mu\left(d_{1}\right)}{d_{1}^{2}}\right)\left(\sum_{d_{2} \mid q_{2}} \frac{\mu\left(d_{2}\right)}{d_{2}^{3}}\right) \\
& \times\left(\sum_{d_{3} \mid q_{3}} \frac{\mu^{2}\left(d_{3}\right)}{d_{3}}\right)\left(\sum_{d_{4} \mid q_{3}} \frac{\mu\left(d_{4}\right) \sigma\left(d_{4}\right)}{d_{4}^{2}}\right) \\
& \times\left(\sum_{d_{5} \mid q_{4}}\left(\frac{-1}{d_{5}}\right) \frac{\mu\left(d_{5}\right)}{d_{5}}\right)\left(\sum_{d_{6} \mid q_{4}}\left(\frac{-1}{d_{6}}\right) \frac{\mu^{2}\left(d_{6}\right)}{d_{6}^{2}}\right) \frac{\varphi^{3}\left(q_{5}\right)}{q_{5}^{3}},
\end{aligned}
$$

where $\sigma\left(d_{4}\right)$ denotes the sum of divisors of $d_{4}$. In particular, if for each prime $p$ dividing $q$ we have $p \mid\left(b^{2} c^{2}-16 a_{1} a_{2} a_{3} a_{4}\right), p \equiv 3(\bmod 4)$ and $\left(\frac{a_{1}}{p}\right)+\left(\frac{a_{2}}{p}\right)+\left(\frac{a_{3}}{p}\right)+$ $\left(\frac{a_{4}}{p}\right)=-4 \cdot\left(\frac{2 c}{p}\right)$ then $N_{q, 4}(\bar{a}, b, c)=\varphi^{3}(q)$.

\section{Poincaré Series}

Let $f \in \mathbb{Z}\left[x_{1}, \ldots, x_{n}\right]$ be a polynomial and let $c_{k}$ denote the number of solutions to the congruence $f\left(x_{1}, \ldots, x_{n}\right) \equiv 0\left(\bmod p^{k}\right)$. The generating function

$$
P_{f}(t)=1+\sum_{k=1}^{\infty} c_{k} t^{k}
$$


is said to be the Poincaré series of $f$. Borevich and Shafarevich [8, p. 47] raised the question of whether $P_{f}(t)$ is always a rational function. Igusa [19] and Denef [12] gave an affirmative answer using completely different methods. Both proofs are nonconstructive and don't show how to express $P_{f}(t)$ as a quotient of two polynomials.

There are, however, certain classes of polynomials for which the corresponding Poincaré series can be computed by elementary means. Goldman $[13,14]$ derived explicit formulas for the Poincaré series associated with strongly nondegenerate forms and with certain algebraic curves. The case of a diagonal polynomial $f\left(x_{1}, \ldots, x_{n}\right)=a_{1} x_{1}^{d_{1}}+\cdots+a_{n} x_{n}^{d_{n}}+c$, where $a_{1}, \ldots, a_{n}, c \in \mathbb{Z}, d_{1}, \ldots, d_{n} \in \mathbb{Z}^{+}$, was treated by Wang [23] (for $c=0$ and $p \nmid a_{1} \cdots a_{n}$ ) and Han [16] (for $p \nmid a_{1} \cdots a_{n} d_{1} \cdots d_{n}$ ), and more recently by Deb [11] (for an arbitrary diagonal polynomial). Recently [4], we calculated explicitly $P_{f}(t)$ for a Markoff-Hurwitz polynomial $f\left(x_{1}, \ldots, x_{n}\right)=x_{1}^{2}+\cdots+x_{n}^{2}-b x_{1} \cdots x_{n}$, where $b \in \mathbb{Z}$ and $n \geq 3$.

Hayes and Nutt [17] presented a further conjecture: $P_{f}(t)$ can be written as $P_{f}(t)=Q_{1}(t) / Q_{2}(t)$, where $Q_{1}(t)$ and $Q_{2}(t)$ are polynomials in $\mathbb{Z}[t]$ (possibly with common factors) and $Q_{2}(t)$ is a product of polynomials of the form $1-p^{m} t^{s}$ with $m, s \in \mathbb{Z}, m \geq 0, s \geq 1$ and $m \leq n s$. They called this assertion the $Q$-conjecture and proved it in a number of cases. Note that the $Q$-conjecture holds for the special classes of polynomials mentioned above.

Now consider the case $f\left(x_{1}, \ldots, x_{n}\right)=a_{1} x_{1}^{2}+\cdots+a_{n} x_{n}^{2}-b x_{1} \cdots x_{n}-c$, where $a_{1}, \ldots, a_{n}, b, c \in \mathbb{Z}, n \geq 3$. Assume that $p>2$ and $p \nmid a_{1} \cdots a_{n} c$. Matching up with our previous notation, we have

$$
P_{f}(t)=1+\sum_{k=1}^{\infty} N_{p^{k}, n}(\bar{a}, b, c) t^{k} .
$$

It is well-known that a power series represents a rational function if and only if the sequence of its coefficients eventually satisfies a linear recurrence relation with constant coefficients. In this case, the denominator is completely determined by the recurrence. The coefficients of the numerator polynomial are determined by the values of the initial terms prior to the recursion. Thus, in view of Corollary 3.6, $P_{f}(t)$ has the form

$$
P_{f}(t)=\frac{R(t)}{\left(1-p^{n-1} t\right)\left(1-\theta p^{n / 2} t\right)}
$$

if $\left(\frac{a_{1}}{p}\right)=\cdots=\left(\frac{a_{n}}{p}\right)=\left(\frac{c(n-2)}{p}\right), b^{2} c^{n-2}=4(n-2)^{n-2} a_{1} \cdots a_{n}$ and $n$ is even,

$$
P_{f}(t)=\frac{R(t)}{\left(1-p^{n-1} t\right)\left(1-p^{n} t^{2}\right)}
$$

if $\left(\frac{a_{1}}{p}\right)=\cdots=\left(\frac{a_{n}}{p}\right)=\left(\frac{c(n-2)}{p}\right), b^{2} c^{n-2}=4(n-2)^{n-2} a_{1} \cdots a_{n}$ and $n$ is odd, and

$$
P_{f}(t)=\frac{R(t)}{1-p^{n-1} t}
$$

otherwise, where $R(t) \in \mathbb{Z}[t]$. We see that the $Q$-conjecture holds in this case. Further, for $b \equiv 0(\bmod p)$, we have $N_{p^{k}, n}(\bar{a}, b, c)=N_{p^{k}, n}(\bar{a}, 0, c)$, in view of the 
remark following Theorem 3.3. Thus, combining the result of Han [16, Theorem 5.3] for diagonal polynomials with (1.3), we deduce that in the case $p \mid b$

$$
\begin{aligned}
R(t) & =1+\left(N_{p, n}(\bar{a}, 0, c)-p^{n-1}\right) t \\
& = \begin{cases}1-\left(\frac{(-1)^{n / 2} a_{1} \cdots a_{n}}{p}\right) p^{(n-2) / 2} t & \text { if } 2 \mid n, \\
1+\left(\frac{(-1)^{(n-1) / 2} a_{1} \cdots a_{n} c}{p}\right) p^{(n-1) / 2} t & \text { if } 2 \nmid n .\end{cases}
\end{aligned}
$$

The results of Sec. 3 allow us to determine $R(t)$ in other cases.

Theorem 5.1. If $\left(\frac{a_{1}}{p}\right)=\cdots=\left(\frac{a_{n}}{p}\right)=\left(\frac{c(n-2)}{p}\right), b^{2} c^{n-2}=4(n-2)^{n-2} a_{1} \cdots a_{n}$ and $n$ is even then

$$
\begin{aligned}
R(t)= & \left(1+\left(N_{p, n}(\bar{a}, b, c)-p^{n-1}\right) t\right)\left(1-\theta p^{n / 2} t\right)+2^{n-1} p^{n-1}(p-1) t^{2} . \\
\text { If }\left(\frac{a_{1}}{p}\right)=\cdots & =\left(\frac{a_{n}}{p}\right)=\left(\frac{c(n-2)}{p}\right), b^{2} c^{n-2}=4(n-2)^{n-2} a_{1} \cdots a_{n} \text { and } n \text { is odd then } \\
R(t) & =\left(1+\left(N_{p, n}(\bar{a}, b, c)-p^{n-1}\right) t\right)\left(1-p^{n} t^{2}\right)+2^{n-1} p^{n-1}(p-1) t^{2} . \\
\text { If }\left(\frac{a_{1}}{p}\right)=\cdots & =\left(\frac{a_{n}}{p}\right)=\left(\frac{c(n-2)}{p}\right), b^{2} c^{n-2} \neq 4(n-2)^{n-2} a_{1} \cdots a_{n} \text { and } r>0 \text { then } \\
R(t)=1 & +\left(N_{p, n}(\bar{a}, b, c)-p^{n-1}\right) t-(-1)^{n(r+1)} \cdot 2^{n-1} \theta^{r+1} p^{[(n(r+1)-1) / 2]} t^{r+1} \\
\times & \left(\frac{\left(b^{2} c^{n-2}-4(n-2)^{n-2} a_{1} \cdots a_{n}\right) / p^{r}}{p}\right)^{n(r+1)} \\
+ & 2^{n-1} p^{n-1}(p-1) t^{2} \cdot \begin{cases}\sum_{j=0}^{r-2} \theta^{j} p^{j n / 2} t^{j} & \text { if } 2 \mid n, \\
\sum_{j=0}^{[(r-2) / 2]} p^{j n} t^{2 j} & \text { if } 2 \nmid n .\end{cases}
\end{aligned}
$$

For all other cases,

$$
R(t)=1+\left(N_{p, n}(\bar{a}, b, c)-p^{n-1}\right) t
$$

\section{Further Generalizations of Markoff-Hurwitz Equations}

In this section, we consider a more general congruence

$$
a_{1} x_{1}^{d_{1}}+\cdots+a_{n} x_{n}^{d_{n}} \equiv b x_{1} \cdots x_{n}+c\left(\bmod p^{k}\right),
$$

where $p>2$ is a prime, $a_{1}, \ldots, a_{n}, b, c$ are integers, $d_{1}, \ldots, d_{n}, k$ are positive integers, $p \nmid a_{1} \cdots a_{n} c d_{1} \cdots d_{n}, n \geq 2$. Assume in addition that at least one of the following conditions holds:

(a) $p \mid b$;

(b) $\operatorname{gcd}\left(d_{j_{1}}, d_{j_{2}}, p-1\right) \nmid\left(\right.$ ind $a_{j_{1}} d_{j_{1}}-$ ind $\left.a_{j_{2}} d_{j_{2}}\right)$ for some $j_{1}, j_{2} \in\{1, \ldots, n\}$; 
(c) $p \mid\left(\left(D / d_{1}\right)+\cdots+\left(D / d_{n}\right)-D\right)$;

(d) $p \nmid\left(\left(D / d_{1}\right)+\cdots+\left(D / d_{n}\right)-D\right)$ and

$$
\operatorname{gcd}\left(d_{j}, p-1\right) \nmid\left(\text { ind } a_{j}\left(\left(D / d_{1}\right)+\cdots+\left(D / d_{n}\right)-D\right)-\operatorname{ind} c D / d_{j}\right)
$$

for some $j \in\{1, \ldots, n\}$;

(e)

$$
\begin{aligned}
& b^{D} c^{\left(D / d_{1}\right)+\cdots+\left(D / d_{n}\right)-D}\left(\frac{D}{d_{1}}\right)^{D / d_{1}} \cdots\left(\frac{D}{d_{n}}\right)^{D / d_{n}} \\
& \quad \neq D^{D}\left(\frac{D}{d_{1}}+\cdots+\frac{D}{d_{n}}-D\right)^{\left(D / d_{1}\right)+\cdots+\left(D / d_{n}\right)-D} a_{1}^{D / d_{1}} \cdots a_{n}^{D / d_{n}},
\end{aligned}
$$

where ind $z$ denotes the index of the integer $z \neq \equiv 0(\bmod p)$ with respect to a fixed primitive root modulo $p$ and $D=\operatorname{lcm}\left[d_{1}, \ldots, d_{n}\right]$.

Throughout this section, $N_{p^{k}, n}(\bar{a}, b, c)$ denotes the number of solutions to (6.1) in $x_{1}, \ldots, x_{n}\left(\bmod p^{k}\right), N_{p^{k}, n}^{*}(\bar{a}, b, c)$ denotes the number of such solutions with $p \nmid x_{1} \cdots x_{n}$, and $N_{p^{k}, n}^{(0)}(\bar{a}, b, c)=N_{p^{k}, n}(\bar{a}, b, c)-N_{p^{k}, n}^{*}(\bar{a}, b, c)$. By the same type of reasoning as in Sec. 3, we can obtain a linear recurrence relation for $N_{p^{k}, n}(\bar{a}, b, c)$ and calculate the corresponding Poincaré series.

For a fixed $k \geq 2$ and $\nu \in\{k-1, k\}$, let $\bar{N}_{p^{\nu}, n}^{(0)}(\bar{a}, b, c)$ and $\bar{N}_{p^{\nu}, n}^{*}(\bar{a}, b, c)$ denote the number of solutions to the system of congruences

$$
\begin{gathered}
a_{1} x_{1}^{d_{1}}+\cdots+a_{n} x_{n}^{d_{n}} \equiv b x_{1} \cdots x_{n}+c\left(\bmod p^{\nu}\right), \\
a_{1} d_{1} x_{1}^{d_{1}-1} \equiv b x_{2} x_{3} \cdots x_{n}\left(\bmod p^{[k / 2]}\right), \\
a_{2} d_{2} x_{2}^{d_{2}-1} \equiv b x_{1} x_{3} \cdots x_{n}\left(\bmod p^{[k / 2]}\right), \\
\vdots \\
a_{n} d_{n} x_{n}^{d_{n}-1} \equiv b x_{1} x_{2} \cdots x_{n-1}\left(\bmod p^{[k / 2]}\right),
\end{gathered}
$$

in $x_{1}, \ldots, x_{n}\left(\bmod p^{\nu}\right)$ with $p \mid x_{1} \cdots x_{n}$ and $p \nmid x_{1} \cdots x_{n}$, respectively. From Lemma 2.1 we deduce that

$$
\begin{aligned}
& N_{p^{k}, n}^{(0)}(\bar{a}, b, c)-p^{n-1} N_{p^{k-1}, n}^{(0)}(\bar{a}, b, c)=\bar{N}_{p^{k}, n}^{(0)}(\bar{a}, b, c)-p^{n-1} \bar{N}_{p^{k-1}, n}^{(0)}(\bar{a}, b, c), \\
& N_{p^{k}, n}^{*}(\bar{a}, b, c)-p^{n-1} N_{p^{k-1}, n}^{*}(\bar{a}, b, c)=\bar{N}_{p^{k}, n}^{*}(\bar{a}, b, c)-p^{n-1} \bar{N}_{p^{k-1}, n}^{*}(\bar{a}, b, c) .
\end{aligned}
$$

By employing the same type of argument as in the proof of Lemma 3.1, we see that $\bar{N}_{p^{k}, n}^{(0)}(\bar{a}, b, c)=\bar{N}_{p^{k-1}, n}^{(0)}(\bar{a}, b, c)=0$, and so

$$
N_{p^{k}, n}^{(0)}(\bar{a}, b, c)=p^{n-1} N_{p^{k-1}, n}^{(0)}(\bar{a}, b, c) \quad \text { for } k \geq 2 .
$$

Next we calculate $N_{p^{k}, n}^{*}(\bar{a}, b, c)-p^{n-1} N_{p^{k-1}, n}^{*}(\bar{a}, b, c)$. When $p \nmid x_{1} \cdots x_{n}$, the system of congruences (6.2) can be rewritten as

$$
\begin{gathered}
a_{1} x_{1}^{d_{1}}+\cdots+a_{n} x_{n}^{d_{n}} \equiv b x_{1} \cdots x_{n}+c\left(\bmod p^{\nu}\right), \\
a_{1} d_{1} x_{1}^{d_{1}} \equiv \cdots \equiv a_{n} d_{n} x_{n}^{d_{n}} \equiv b x_{1} \cdots x_{n}\left(\bmod p^{[k / 2]}\right) .
\end{gathered}
$$


Observe that (6.3) yields the congruences

$$
\left(\frac{D}{d_{1}}+\cdots+\frac{D}{d_{n}}-D\right) a_{j} x_{j}^{d_{j}} \equiv c \cdot \frac{D}{d_{j}}\left(\bmod p^{[k / 2]}\right), \quad 1 \leq j \leq n .
$$

It follows easily that if at least one of the conditions (a)-(d) holds then $\bar{N}_{p^{k}, n}^{*}(\bar{a}, b, c)=\bar{N}_{p^{k-1}, n}^{*}(\bar{a}, b, c)=0$, and so $N_{p^{k}, n}^{*}(\bar{a}, b, c)=p^{n-1}{N_{p^{k-1}, n}^{*}}_{a}(\bar{a}, b, c)$ for $k \geq 2$.

Now suppose that none of (a)-(d) holds. Hence the condition (e) holds. If $\bar{N}_{p^{k-1}, n}(\bar{a}, b, c)=0$ then we are done. Assume that $\bar{N}_{p^{k-1}, n}(\bar{a}, b, c) \neq 0$ and $x_{1}, \ldots, x_{n}$ are integers with $p \nmid x_{1} \cdots x_{n}$ satisfying (6.2). Then

$$
\left(a_{1} x_{1}^{d_{1}}+\cdots+a_{n} x_{n}^{d_{n}}-c\right)^{D} \equiv b^{D} x_{1}^{D} \cdots x_{n}^{D} \quad\left(\bmod p^{k-1}\right) .
$$

Multiplying both sides by $\left(\frac{D}{d_{1}}+\cdots+\frac{D}{d_{n}}-D\right)^{\left(D / d_{1}\right)+\cdots+\left(D / d_{n}\right)} a_{1}^{D / d_{1}} \cdots a_{n}^{D / d_{n}}$, we obtain

$$
\begin{aligned}
\left(\frac{D}{d_{1}}+\right. & \left.\cdots+\frac{D}{d_{n}}-D\right)^{\left(D / d_{1}\right)+\cdots+\left(D / d_{n}\right)-D} a_{1}^{D / d_{1}} \cdots a_{n}^{D / d_{n}} \\
& \times\left(\sum_{j=1}^{n}\left(\left(\frac{D}{d_{1}}+\cdots+\frac{D}{d_{n}}-D\right) a_{j} x_{j}^{d_{j}}-c \cdot \frac{D}{d_{j}}\right)+c D\right)^{D} \\
\equiv & b^{D} \prod_{j=1}^{n}\left(\left(\frac{D}{d_{1}}+\cdots+\frac{D}{d_{n}}-D\right)^{D / d_{j}} a_{j}^{D / d_{j}} x_{j}^{D}\right)\left(\bmod p^{k-1}\right) .
\end{aligned}
$$

Further, using (6.4), we find that

$$
\begin{gathered}
D^{D}\left(\frac{D}{d_{1}}+\cdots+\frac{D}{d_{n}}-D\right)^{\left(D / d_{1}\right)+\cdots+\left(D / d_{n}\right)-D} a_{1}^{D / d_{1}} \cdots a_{n}^{D / d_{n}} \\
\equiv b^{D} c^{\left(D / d_{1}\right)+\cdots+\left(D / d_{n}\right)-D}\left(\frac{D}{d_{1}}\right)^{D / d_{1}} \cdots\left(\frac{D}{d_{n}}\right)^{D / d_{n}}\left(\bmod p^{[k / 2]}\right) .
\end{gathered}
$$

In view of the condition (e), the latter is not true if $k$ is sufficiently large. Thus $\bar{N}_{p^{k}, n}^{*}(\bar{a}, b, c)=\bar{N}_{p^{k-1}, n}^{*}(\bar{a}, b, c)=0$ and $N_{p^{k}, n}^{*}(\bar{a}, b, c)=p^{n-1} N_{p^{k-1}, n}^{*}(\bar{a}, b, c)$ for sufficiently large $k$.

The results may be summarized as follows.

Theorem 6.1. For sufficiently large $k$

$$
N_{p^{k}, n}(\bar{a}, b, c)=p^{n-1} N_{p^{k-1}, n}(\bar{a}, b, c) .
$$

In particular, if at least one of the conditions $(a)-(d)$ holds then the number of solutions satisfies the above recurrence relation for all $k \geq 2$.

Taking into account the fact that $N_{p, n}(\bar{a}, b, c)=p^{n-1}$ if $\operatorname{gcd}\left(d_{j}, p-1\right)=1$ for some $j$ and $p \mid b$, we obtain the following corollary. 
Corollary 6.2. Let $p>2$ be a prime and

$$
f\left(x_{1}, \ldots, x_{n}\right)=a_{1} x_{1}^{d_{1}}+\cdots+a_{n} x_{n}^{d_{n}}-b x_{1} \cdots x_{n}-c,
$$

where the integers $a_{1}, \ldots, a_{n}, b, c, d_{1}, \ldots, d_{n}$ satisfy the above conditions. Then the Poincaré series $P_{f}(t)$ is a rational function of the form

$$
P_{f}(t)=\frac{R(t)}{1-p^{n-1} t},
$$

where $R(t) \in \mathbb{Z}[t]$; in particular, if $\operatorname{gcd}\left(d_{j}, p-1\right)=1$ for some $j$ and $p \mid b$ then $R(t)=1$.

Finally, we notice that the $Q$-conjecture of Hayes and Nutt [17] holds for this class of polynomials.

\section{References}

[1] T. M. Apostol, Introduction to Analytic Number Theory (Springer-Verlag, New York, 1976).

[2] I. Baoulina, On the problem of explicit evaluation of the number of solutions of the equation $a_{1} x_{1}^{2}+\cdots+a_{n} x_{n}^{2}=b x_{1} \cdots x_{n}$ in a finite field, in Current Trends in Number Theory, eds. S. D. Adhikari, S. A. Katre and B. Ramakrishnan (Hindustan Book Agency, New Delhi, 2002), pp. 27-37.

[3] _ Generalizations of the Markoff-Hurwitz equations over finite fields, J. Number Theory 118 (2006) 31-52.

[4] _ On Markoff-Hurwitz equations over residue class rings, Int. J. Number Theory 10 (2014) 421-454.

[5] A. Baragar, The Markoff equation and equations of Hurwitz, Ph.D. thesis, Brown University (1991).

[6] _ The Markoff-Hurwitz equations over number fields, Rocky Mountain J. Math. 35 (2005) 695-712.

[7] B. C. Berndt, R. J. Evans and K. S. Williams, Gauss and Jacobi Sums (WileyInterscience, New York, 1998).

[8] Z. I. Borevich and I. R. Shafarevich, Number Theory (Academic Press, New York, 1966).

[9] L. Carlitz, Certain special equations in a finite field, Monatsh. Math. 58 (1954) 5-12.

[10] E. Cohen, Rings of arithmetic functions. II. The number of solutions of quadratic congruences, Duke Math. J. 21 (1954) 9-28.

[11] D. Deb, Diagonal forms and the rationality of the Poincaré series, Ph.D. thesis, University of Kentucky (2010).

[12] J. Denef, The rationality of the Poincaré series associated to the $p$-adic points on a variety, Invent. Math. 77 (1984) 1-23.

[13] J. R. Goldman, Numbers of solutions of congruences: Poincaré series for strongly nondegenerate forms, Proc. Amer. Math. Soc. 87 (1983) 586-590.

[14] _ Numbers of solutions of congruences: Poincaré series for algebraic curves, Adv. in Math. 62 (1986) 68-83.

[15] S. J. Gurak, Kloosterman sums for prime powers in $P$-adic fields, J. Théor. Nombres Bordeaux 21 (2009) 175-201.

[16] Q. Han, Numbers of solutions of congruences and rationality of generating functions, Finite Fields Appl. 5 (1999) 266-284. 
[17] D. Hayes and M. D. Nutt, Reflective functions on p-adic fields, Acta Arith. 40 (1982) $229-248$.

[18] A. Hurwitz, Über eine Aufgabe der unbestimmten analysis, Arch. Math. Phys. 3 (1907) 185-196.

[19] J. Igusa, Complex powers and asymptotic expansions. II. Asymptotic expansions, J. Reine Angew. Math. 278/279 (1975) 307-321.

[20] C. Jordan, Sur les congruences du second degré, C. R. Acad. Sci. Paris 62 (1866) 687-690.

[21] A. A. Markoff, Sur les formes quadratiques binaires indéfinies, Math. Ann. 17 (1880) 379-399.

[22] J. H. Silverman, The Markoff equation $X^{2}+Y^{2}+Z^{2}=a X Y Z$ over quadratic imaginary fields, J. Number Theory 35 (1990) 72-104.

[23] J. Wang, On Poincaré series for diagonal forms, Proc. Amer. Math. Soc. 116 (1992) 607-611. 